\title{
Multi-View Images 3D Reconstruction based on Spatial Geometric Constraint
}

\author{
Haibo Liu \\ PLA 91550 Unit 94, Dalian, 116023, China \\ hunter24099@163.com
}

Keywords: 3D Reconstruction; PMVS; Spatial Geometric Constraint

\begin{abstract}
The PMVS algorithm due to lacking of geometric constraint during expanding spare points to dense points cloud, resulting in error vertices which are generated from Poisson surface reconstruction. In This paper we consider facets around the expanding one at the same time, then detect the outlier vertices of triangle meshes according to the $\mathrm{k}$ Nearest Neighbor and remove outliers. Experimental results demonstrate the effectiveness and accuracy of the algorithm.
\end{abstract}

\section{Introduction}

Multi-view 3D reconstruction techniques is to study how the two-dimensional information of the object to obtain 3D information of objects in space, and ultimately to obtain a 3D model of the object space. Many scholars and researchers have done a lot of work in the 3D reconstruction algorithm. According to the object model algorithm, algorithms can be summarized as follows: voxel-based algorithms [1], the algorithm needs to know contains a scene boundary, and its accuracy receptor pixel grid resolution limit; based deformable polygon mesh algorithms [2], the algorithm requires a good initial value to initialize the corresponding optimization process, which it limits its application; multiple depth map based algorithms [3], the algorithm is more flexible, but need to be combined into a depth map for each 3D model; algorithm facets based [5], by plane piece set to represent the surface, this algorithm is simple and effective. Article [6] proposed a multi-view stereo vision PMVS algorithm based on facet, the algorithm is better reconstruction algorithm. In this paper PMVS algorithm [6] on the basis of algorithms in less time to make improvements extended by adding geometric constraints of space, so that reconstruction tends to smooth the surface, and surface reconstruction algorithm Poisson [5] to obtain a 3D model of the object surface, the generated error facet vertices do outlier detection, filter generated by these outliers vertices errors facets to give a 3D model showing the surface of the real object.

\section{PMVS algorithm Fundamentals}

PMVS algorithm can be divided into three steps: matching, extensions and filters, which extend and 2-step filtration repeated $\mathrm{n}$ times (experiment 3 times).The algorithm to reconstruct a 3D point rectangular facet, said facet is the center point of the 3D direction of Facet for points. To facilitate the description, some definitions algorithm is given first:

Get the picture provided to the subject for the set $\operatorname{Im}=\left\{I_{i} \mid i=1,2, \ldots, n\right\}, O\left(I_{i}\right)$ showing the $i$ image corresponding to the camera's optical center. PMVS reconstruction algorithm to judge whether the results of the standard is to rebuild dense facet can cover the entire surface of the object, firstly the image $I_{i}$ is divided into set of image blocks, each image block is represented as $C_{i}(x, y), \mathrm{x}, \mathrm{y}$ is the image block index. If all image blocks contain at least one facet reconstruction projection surface is considered full coverage. For a facet $f$ with the following definitions:

$c(f)$ : Center of the facet $f$; $n(f)$ : Facet $f$ unit normal; $R(f)$ : Facet $f$ reference pictures; $V(f)$ : Facet $f$ meet Angle constraint visible image set; $g(f)$ : Grayscale difference function; $V^{*}(f): V(f)$ Gray Consistency satisfying set of images; $g^{*}(f): V^{*}(f)$ Alternative $V(f)$ obtain different gradation function; $C(f)$ : A set of adjacent image blocks facet $f$ visible in the picture; $Q(x, y)$ : Images projected onto the block $C(x, y)$ in all facets set. 
Before reconstruction need to determine the parameters of the inside and outside of the camera, and then get the unipolar constraint relationship among images, the reconstruction process can be divided into three steps: feature matching, extension, and filtering.

\section{Algorithm based on improved spatial geometric constraint}

PMVS algorithm extended from sparse to dense facets, only the current facet $f$ information to initialize the extended facet, and no other facets around the utilization of information, so the overall result of the extension connection is bad, especially in the presence of surface gap junction depth change is not smooth enough. To solve these problems, we propose taking into account the impact of the extended facet around the adjacent, so that reconstruction results smoother. Specific improvements as follows:

Step1: In each image block $C_{i}(x, y)$ of $c(p)$, in the extension neighboring facet $f^{\prime}$ of current facet $f$, and following the direction $p p^{\prime}$ and finding another recent facet $f^{\prime \prime}$, and set from the current facet $f$ its vertical distance $a$ the horizontal distance $b$. In addition, line between the optical center $O\left(I_{i}\right)$ and $C_{i}(x, y)$ center, intersection with facet $f$ is point $f_{0}$. $f f_{0}$ denotes the distance between $f^{\prime}$ and $f$ note as $l$, so the distance between $f^{\prime \prime}$ and $f^{\prime}$ is $b-l$.

Step2: At initialization extended facet normal $n\left(f^{\prime}\right)$, and considering the impact of $n(f)$ and $n\left(f^{\prime \prime}\right)$, their weights are $(b-l) / b$ and $l / b$, set the direction angle of $n(f)$ is $\alpha$, direction angle of $n\left(f^{\prime \prime}\right)$ is $\beta$, direction angle of $n\left(f^{\prime}\right)$ is $\gamma$. However, if distance between $f$ and $f^{\prime}$, we use $n(f)$ to initialize $n\left(p^{\prime}\right)$ :

$$
\gamma= \begin{cases}\gamma=\frac{b-l}{b} \cdot \alpha+\frac{l}{b} \cdot \beta & (a, b) \in \Delta(a, b) \\ \alpha & \text { else }\end{cases}
$$

Step3: Definite $\Delta(\alpha, \beta)=\left\{(\alpha, \beta) \mid \alpha \leq l_{\alpha}, \mathrm{b} \leq l_{b}, 0.3<a / b<2\right\}$ to describe the relationship between $f$ and $f^{\prime \prime}$.

Step4: Extended facet visible image set $V\left(f^{\prime}\right)$ has two parts, all visible pictures $V(f)$ of current sheet is part one, the most recent facets visible image set $V\left(f^{\prime \prime}\right)$ is another part, which will be in line with the angle range constraint pictures added to the extended facet visible picture sets, as follows:

$$
V\left(f^{\prime}\right)=V(f)+\left\{I \mid \frac{n\left(f^{\prime}\right) \cdot c\left(f^{\prime}\right) O(I)}{\left|c\left(f^{\prime}\right) O(I)\right|}>\cos (\tau), I \in V\left(f^{\prime \prime}\right)\right\}
$$

Wherein, $\tau$ is visible angle threshold, set $\tau=\pi / 3$.

Step5: Algorithm in determining the center of the facet $c\left(f^{\prime}\right)$, maintain its optical center by the reference camera image and block the line of centers located on a straight line, and then finally determined by the vertical distance between $c\left(f^{\prime}\right)$ and $f$ in its the plane. Definite vertical distance between $c\left(f^{\prime}\right)$ and $f$ in it's the plane:

$$
h= \begin{cases}1 & (a, b) \in \Delta(a, b) \\ 0 & (a, b) \in \Delta(a, b)\end{cases}
$$

Step6: Initialization $n\left(f^{\prime}\right), c\left(f^{\prime}\right)$ and $V\left(f^{\prime}\right)$, and after, the conjugate gradient methods iterative adjustment extended position and direction of the facet $f^{\prime}$, after convergence finally get the facet $f^{\prime}$ is extended out of the new facet, sparse facet set of all facets do the same extended operation until expanded into a dense facet set up.

\section{Outlier detection based on distance}

Article [8] proposed a new outlier detection algorithm based on distance: with a point and it $D_{k}\left(f^{\prime}\right)$ represents the distance between point $f$ and the $k$ nearest neighbor, given $D$ dimensional space that contains a set of $N$ data points. Natural number parameters $n$ and $k$, if satisfy the condition $D_{k}\left(f^{\prime}\right)>D_{k}(f)$, the point $f^{\prime}$ of no more than $n-1$, then called $f$ the $D_{k}(f)$ outliers. If the data points rank according to their $D_{k}(f)$, then the first $n$ objects to be 
viewed as outliers.

But the parameter $n$ is not well determined, to solve this problem is proposed based on the distance and the outlier detection, eliminating the user requirements of setting the parameters of the, but increases the computation time. So in this article points are sorted according to their $D_{k}(f)$ ranking, and calculate the average distance $\bar{d}$, set the threshold value $\lambda=3 \bar{d}$, outliers are considered greater than this threshold, all the vertices are, expressed as follows:

$$
\operatorname{Flag}(f)= \begin{cases}0 & k-\operatorname{distance}(f)<\lambda \\ 1 & k \text {-distance }(f)>\lambda\end{cases}
$$

Where 0 represents the vertex normal points; 1 represents the apex of outliers. For this paper, measure the distance of the Euclidean distance. The outlier removal where the facet is finally get the real 3D model of the object's surface.

\section{Test results and analysis}

Our test environment is Intel(R)Core(TM) i7 CPU $2.80 \mathrm{GHz}, 4 \mathrm{~GB}$ memory, Windows7 operating system。Visual $\mathrm{C}++$ is used for implementation. In this paper, the experiment threshold $l_{\alpha}=4 l, l_{b}=3 l$ is calculated for each constant. Threshold value $3 d^{\prime}$ and $d^{\prime}$ are the average distance from the vertex.

Experiment with the real objects and scenes shot from multiple angles a picture sequence as the original input data, then each of these data reconstruction algorithm used herein PMVS algorithm and improved and improved into two parts: (1) extended PMVS algorithm added space geometric constraint; (2) add outlier detection into the Poisson surface reconstruction results to find the error vertex, and then filter facets caused by the wrong vertex, and finally get the results.

Experimental data obtained by a digital camera 9 image sequence which is the original picture format of $4288 * 2848$ pixels (Figure 1), and then by the sift feature matching calculated each picture corresponding projection matrix. The results algorithm (Figure 2) after PMVS algorithm and improved by the article, as well as the results of the reconstruction and removal of Poisson results (Figure 3) constitutes an error after the facet.
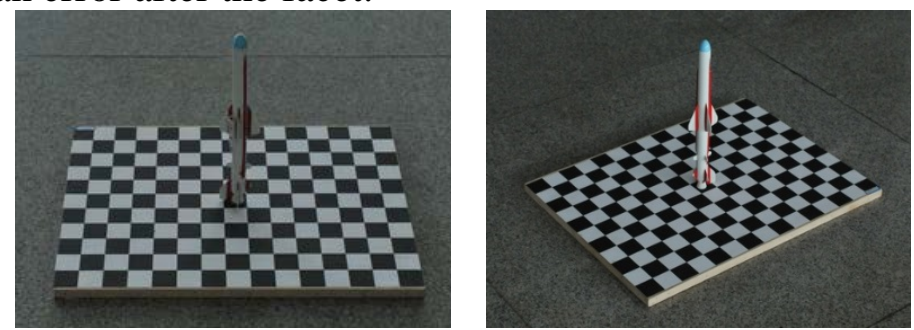

Fig.1. Original pictures

The original PMVS algorithm, a new facet in the extended sheet with the current facet the plane to determine the new position of the center of the dough piece, using the current method to determine the new facet, trying to make the new facet is consistent with the current facet, but it ignores the impact on the surrounding other facets of the new facet, so that changes in the area often have deep stratification.

By considering the left and right side pieces on the current extension of the dough piece, which joined the geometry of space constraints, so that the surface is more smooth distribution sheet, shown in Figure 2. While extending to obtain the number of facets is also a corresponding increase in the first group test of the original PMVS algorithm facet number is 30910, the improved get 32402. Based on 3D point cloud PMVS reconstruction obtained using Poisson surface reconstruction algorithms can reconstruct a surface model of a closed, and this model is closed, therefore, the algorithm will generate some dough piece does not actually exist in the back surface to form a closed surfaces, vertex detecting outlier article by adding, to filter out those errors generated vertex, to obtain the true surface model represented in Figure 3. Improved results are shown in Table 1. 


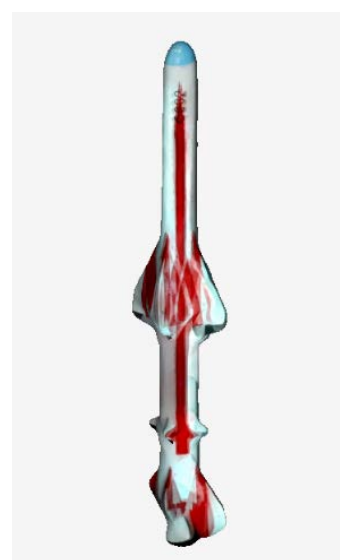

Fig.2. Improved PWVS method

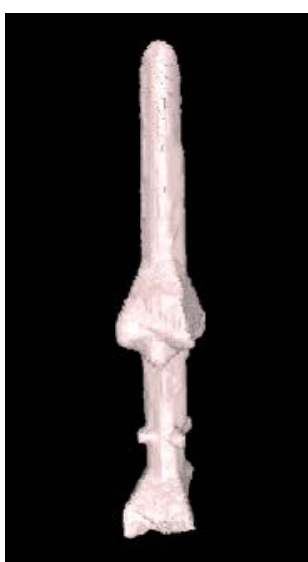

Fig.3. results after outliers removal
Table 1 Surface reconstruction result before and after filtering outliers

\begin{tabular}{ccc}
\hline & Vertex number & Facet number \\
\hline Improve before & 32402 & 65239 \\
\hline Improve after & 31086 & 62368 \\
\hline
\end{tabular}

Experimental results show that the improved results of the 3D reconstruction, the total number of facets increase about 5\%. Poisson errors can be filtered through outliers detection, the filter rate is about $4 \%$.

\section{Conclusion}

PMVS algorithm for high-resolution textures rich object or scene reconstruction with good results, but the corresponding low resolution and reconstruction of objects without texture or textures duplicate the effect is not ideal, especially in the area of in-depth changes, the resulting film will face there is a gap, so that the reconstruction surface looks smooth enough, so this article by adding geometric constraints of space to adjust the position and direction of the dough piece, so as to ensure the reconstruction of 3D surface of the sheet as continuous and smooth, but does not appear there is a gap of stratification, thereby improving the accuracy of reconstruction. In addition, the paper Poisson surface reconstruction results found by the apex outlier detection and removal of vertices, in addition to the error facet to finally obtain a 3D model of the object represents the real surface.

\section{References}

[1] Pons J P Keriven R. Faugeras O. Multi-view Stereo Re-construction and Scene Flow Estimation with a Global Image Based Matching Score [J]. International Journal of Computer Vision, 2007, 2(72):179-193.

[2] Esteban C H Schmitt F. Silhouette and Stereo Fusion for 3D Object Modeling [J]. Computer Vision and Image Understanding, 2004, 3(96):367-392.

[3] Goesele M Curless B. Seitz S. Multi-view Stereo Revisited [C]. Proceedings of IEEE Conference on Computer Vision and Pattern Recognition. New York, USA:IEEE Computer Society, 2006:2402-2409.

[4] Furukawa Y Ponce J. Accurate, Dense, and Robust Multi-view Stereopsis [J]. IEEE Transactions on Pattern Analysis and Machine Intelligence, 2010, 8(32):1362-1372.

[5] Kazhdan M Bolitho M. Hoppe H. Poisson Surface Recon- struction [C]. Proceedings of the 4th Eurographics Symposium on Geometry Processing. Cagliari. Cagliari, Italy:Eurographics Association, 2006:61-70.

[6] Ramaswamy S Rastogi R. Kyuseok S. Efficient Algorithms for Mining Outliers from Large Data Sets [C]. Proceedings of ACMSIGMOD International Conference on Management of Data. allas, USA:ACM Press, 2000:427-438. 